\title{
Hemorragia digestiva baja masiva en pacientes con enfemedad de Crohn
}

\author{
M. Barreiro de Acosta, S. Seijo Ríos y J. E. Domínguez Muñoz \\ Servicio de Aparato Digestivo. Hospital Clínico Universitario. Santiago de Compostela, A Coruña
}

\section{RESUMEN}

Objetivo: analizar la frecuencia de las hemorragias masivas en la EC e intentar determinar sus potenciales factores de riesgo (ambientales, propios de la enfermedad y genéticos).

Material y métodos: se estudió de manera retrospectiva una cohorte de 174 pacientes -103 mujeres (59\%) y 71 hombres (41\%), edad media de 37 años- con EC, analizándose las hemorragias digestivas masivas que se habían producido en relación con su enfermedad. Se revisaron asimismo potenciales factores de riesgo como hábito tabáquico, localización de la enfermedad y presencia de mutaciones genéticas en CARD15, RTL-4 y CD14, entre otros.

Resultados: tres pacientes $(1,7 \%)$ presentaron una hemorragia digestiva masiva que precisó intervención quirúrgica para su resolución. Esta indicación de cirugía supone el 3,4\% de las cirugías en relación con la EC. Todos los pacientes eran jóvenes y su enfermedad seguía un patrón inflamatorio y estaba localizada en el íleon. No se ha evidenciado asociación estadísticamente significativa entre las hemorragias digestivas masivas y los potenciales factores de riesgo analizados.

Conclusiones: la hemorragia digestiva baja masiva es una complicación poco común, aunque grave de la EC. Se trata habitualmente de pacientes con patrón inflamatorio de la enfermedad y afectación ileal. Para su diagnóstico resulta clave la asociación de endoscopia y arteriografía, y su resolución suele ser quirúrgica.

Palabras clave: Enfermedad de Crohn. Hemorragia. Factores predictivos.

\begin{abstract}
Objective: we aimed at evaluating the frequency of acute severe bleeding in $\mathrm{CD}$ and its potential association to some risk factors, including clinical features of $\mathrm{CD}$, environmental factors, and genetic alterations.

Material and methods: 174 consecutive patients with CD (103 female (59\%) and 71 men (41\%), with a mean age of 37 years) were included. We analyzed all major acute lower gastrointestinal (GI) hemorrhage related to $\mathrm{CD}$. Potential risk factors like smoking, site of disease, and presence of gene mutations in CARD15, TLR-4, and CD14 were also analyzed.

Results: three patients (1.7\%) suffered from severe acute lower GI bleeding. All patients required surgery to resolve their hemorrhage, and this indication represented $3.4 \%$ of all surgical procedures related to $\mathrm{CD}$. All three patients were young ( $<25$ years) and suffered ileal CD with inflammatory pattern (L1-B1 in the Vienna Classification). No relationship was found between acute bleeding and any of the potential risk factors evaluated.

Conclusions: acute severe GI bleeding is a rare, but severe complication in CD patients, and presents mainly in patients with inflammatory ileal disease. An association of endoscopy and arteriography is necessary for diagnosis. Urgent surgery is usually required in these patients.
\end{abstract}

Barreiro de Acosta M, Seijo Ríos S, Domínguez Muñoz JE. Hemorragia digestiva baja masiva en pacientes con enfermedad de Crohn. Rev Esp Enferm Dig 2007; 99: 388-391.

Recibido: 05-12-06.

Aceptado: 11-04-07.

Correspondencia: Manuel Barreiro de Acosta. Servicio de Aparato Digestivo. Hospital Clínico Universitario. C/ Choupana, s/n. 15706 Santiago de Compostela, A Coruña. e-mail: manubarreiro@hotmail.com

\section{INTRODUCCIÓN}

El sangrado gastrointestinal bajo se ha descrito en numerosas ocasiones como un síntoma o un signo clínico habitual en los pacientes con enfermedad inflamatoria in- 
testinal (EII), sin embargo la gran mayoría, por no decir la casi totalidad de los casos, se refiere a pacientes con colitis ulcerosa (CU). En la enfermedad de Crohn (EC) es más rara esta presentación, resultando extremadamente inusual los casos en los que el sangrado es masivo, con inestabilidad hemodinámica $\mathrm{y}$, que frecuentemente requiere cirugía para su resolución.

Las series muestran que la presencia de sangrado masivo en pacientes con EC se sitúa entre el 0,6 y el $2 \%$ de los casos, siendo el porcentaje aún menor cuando sólo se contabilizan los pacientes que requieren cirugía para su resolución $(1,2)$. Salvo excepciones, los sangrados masivos en EC se han presentado como casos clínicos aislados, en la mayoría de los casos no se han realizado referencias a la población estudiada, ni se han analizado probables causas o factores de riesgo que pudiesen estar asociados a estas hemorragias masivas $(3,4)$.

La enfermedad de Crohn es una entidad que en múltiples ocasiones requiere cirugía durante su curso evolutivo, siendo las intervenciones más frecuentes las resecciones ileales en pacientes con un patrón evolutivo estenosante (B2 de la clasificación de Viena) (5) y la cirugía de las fístulas. Sin embargo no se conoce el porcentaje de pacientes cuya causa de intervención sea la presencia de hemorragia masiva.

El objetivo de este estudio es analizar la frecuencia de las hemorragias masivas en una cohorte de pacientes correctamente estructurada dentro de una Unidad de Enfermedad Inflamatoria Intestinal e intentar averiguar potenciales factores predictivos, tanto ambientales, propios de la enfermedad, como genéticos que puedan ser útiles a la hora de predecir el riesgo de desarrollar esta grave manifestación de la enfermedad.

\section{MATERIAL Y MÉTODOS}

Se estudiaron de manera retrospectiva 174 pacientes con enfermedad de Crohn -103 mujeres (59\%) y 71 hombres (41\%), edad media de 37 años, un rango entre $17 \mathrm{y}$ 76 y un tiempo medio de seguimiento de su enfermedad de 7 años y medio- a seguimiento en la Unidad de Enfermedad Inflamatoria Intestinal del Servicio de Aparato Digestivo del Hospital Clínico Universitario de Santiago de Compostela. El diagnóstico de EC se realizó a través de métodos clínicos, endoscópicos, radiológicos e histológicos, siendo todos ellos revisados por el mismo especialista en Aparato Digestivo para confirmar la EC y evitar distintos criterios de selección.

Se definió como hemorragia digestiva masiva la presencia de hematoquecia o melenas con inestabilidad hemodinámica (hipotensión o alteración de los signos vitales) y/o descenso agudo de $2 \mathrm{~g} / \mathrm{dl}$ en la hemoglobina en comparación con la basal (1).

Todos los pacientes fueron estratificados por edad y sexo. Asimismo se estudiaron factores ambientales como los antecedentes familiares de EC (familiares de primer grado), hábitos tabáquicos, así como el origen rural o urbano. También se han evaluado aspectos relacionados con la propia enfermedad como los distintos fenotipos en relación con la clasificación de Viena (localización, edad de presentación y comportamiento evolutivo), la presencia de manifestaciones extraintestinales y, los antecedentes quirúrgicos de estos pacientes en relación con su EC. Con la hipótesis de que la predisposición genética pudiese tener valor pronóstico para el desarrollo de hemorragia masiva también se evaluó la presencia de alguna de las tres principales mutaciones del gen NOD2/CARD15 (R702W, G908R y 1007fs) (6) así como la presencia de mutaciones asociada a un polimorfismo en el gen que codifica los receptores toll-like tipo 4 (TLR4) (Asp299Gly) y una mutación en un polimorfismo del gen CD14 (T/C 260), ambas cercanas al gen NOD2 e implicadas en la fisiopatología de la EC $(7,8)$.

Los resultados han sido expresados en porcentajes; las asociaciones entre la presencia de hemorragia masiva y sus potenciales factores predictivos se estudió mediante test de chi-cuadrado o test exacto de Fisher. Se consideró estadísticamente significativo un valor de $\mathrm{p}<0,05$. En caso de que existiese más de un factor asociado significativamente al desarrollo de hemorragia masiva se realizaría análisis multivariante mediante regresión logística no condicional.

\section{RESULTADOS}

Tres pacientes $(1,7 \%)$ presentaron una hemorragia digestiva masiva. La abundante presencia de sangre en el colon impidió alcanzar íleon en los tres casos. Tras confirmarse el punto de sangrado mediante arteriografía, ileal en todos ellos, la resolución del mismo fue posible únicamente de forma quirúrgica. Los tres pacientes (dos varones y una mujer) eran de edad joven (menores de 28 años) y con un diagnóstico relativamente reciente de su enfermedad (media de 2 años). Los tres eran de origen urbano, dos de ellos fumadores y en cuanto al componente familiar uno de ellos tiene un familiar de primer grado (madre) con EC.

En cuanto a las características de su enfermedad, los tres pacientes tenían un patrón inflamatorio (B1). Dos de ellos presentaban localización ileal pura, mientras que otro también tenía afectación del colon derecho. Ninguno de los pacientes había presentado manifestaciones extraintestinales y tampoco ninguno de ellos había sido previamente intervenido quirúrgicamente en relación con su enfermedad. Dos de los tres pacientes estaban recibiendo tratamiento inmunosupresor (azatioprina) y uno de ellos había recibido tratamiento con infliximab por haber desarollado previamente córtico-dependencia.

En cuanto a la presencia de mutaciones genéticas, solamente un paciente presentaba mutaciones de gen NOD2 (en concreto es heterocigoto para R702W) y, ese mismo paciente presentaba mutaciones en CD14 
(T/C 260). Ninguno presentaba alteraciones genéticas en TLR4 (Asp299Gly).

La característica común de estos tres casos es que se resolvieron mediante arteriografía seguida de resección ileal, tras no ser posible la resolución endoscópica, presentando una buena evolución clínica, tanto en el postoperatorio como en los meses posteriores.

Las características epidemiológicas, clínicas y genéticas de los tres pacientes que han presentado hemorragia digestiva masiva se muestran en la tabla I.

El escaso número de pacientes $(n=3)$ con hemorragia digestiva masiva ha limitado enormemente la aplicación e interpretación de los estudios estadísticos en relación con los probables factores predictivos. No se ha encontrado asociación significativa entre el desarrollo de hemorragia digestiva masiva y factores ambientales, propios de la EC o genéticos. Solamente se ha observado una tendencia a la asociación de la hemorragia con el origen urbano de los 3 pacientes $(\mathrm{p}=0,06)$; sobre todo teniendo en cuenta que nuestra cohorte de pacientes es mayoritariamente rural $(57 \%)$.

De los 174 pacientes estudiados, 88 (50,5\%) han sido intervenidos quirúrgicamente. Esto significa que en nuestra población de estudio el 3,4\% de las intervenciones quirúrgicas en pacientes con EC son debidas a hemorragias digestivas masivas.

\section{DISCUSIÓN}

Entre las complicaciones intestinales de la EC, la hemorragia digestiva baja masiva es poco frecuente, siendo claramente superada por fístulas, abscesos y estenosis. En con-

\begin{tabular}{|c|c|c|c|}
\hline & Caso 1 & Caso 2 & Caso 3 \\
\hline Sexo & Mujer & Varón & Varón \\
\hline Edad & 25 años & 28 años & 22 años \\
\hline Origen & Urbano & Urbano & Urbano \\
\hline $\begin{array}{c}\text { Antecedentes } \\
\text { familiares Ell }\end{array}$ & $\begin{array}{l}\text { Presente (madre } \\
\text { con EC) }\end{array}$ & Ausente & Ausente \\
\hline Hábito tabáquico & Sí & No & Sí \\
\hline $\begin{array}{l}\text { Localización enfer- } \\
\text { medad de Crohn }\end{array}$ & Íleon terminal & $\begin{array}{l}\text { Íleon terminal } \\
\text { Colon derecho }\end{array}$ & Íleon terminal \\
\hline Patrón & Inflamatorio & Inflamatorio & Inflamatorio \\
\hline $\begin{array}{l}\text { Manifestaciones } \\
\text { extraintestinales }\end{array}$ & Ausentes & Ausentes & Ausentes \\
\hline $\begin{array}{l}\text { Tratamiento } \\
\text { inmunosupresor }\end{array}$ & No & $\begin{array}{l}\text { Azatioprina } \\
\text { Infliximab }\end{array}$ & Azatioprina \\
\hline Mutación NOD2 & No & No & Heterocigoto R702W \\
\hline Mutación 14-260 & No & No & Presente \\
\hline Mutación TLR-299 & No & No & No \\
\hline \multirow[t]{3}{*}{ Arteriografía } & Anormalidad vas- & Anormalidad vas- & Irregularidad y \\
\hline & cular en íleon pélvico & cular en íleon terminal. & - aumento de cali- \\
\hline & $\begin{array}{c}\text { Sangrado activo } \\
\text { Resección }\end{array}$ & $\begin{array}{l}\text { Sangrado activo } \\
\text { Resección }\end{array}$ & bre de vasos en \\
\hline quirúrgica & ileocecal & $\begin{array}{l}\text { Reseccion } \\
\text { ileocecal }\end{array}$ & $\begin{array}{l}\text { Reseccion } \\
\text { ileocecal }\end{array}$ \\
\hline
\end{tabular}

creto, en nuestra población se ha presentado en el 1,7\% de los casos, siendo nuestros resultados similares o ligeramente superiores a otras poblaciones estudiadas $(1,2,9)$. Cabe destacar que estudios previos incluyen un número superior de pacientes era superior al nuestro, en especial el realizado por el grupo de Mount Sinai (2) en el que en más de 1.200 pacientes con EC reportan un 1,7\% de pacientes con hemorragias masivas. En cambio, en estudios en los que se recoge un menor número de pacientes que en el nuestro se han descrito porcentajes superiores de hemorragias masivas (entre el 3 y el 5\%) $(10,11)$. Nuestra población de estudio es una cohorte de pacientes relativamente joven, apenas superan los 7 años de media de enfermedad, mientras que en las poblaciones con más pacientes esta media resulta mayor. Este hecho, dado que la mayoría de las hemorragias masivas se presentan en pacientes con una media de tiempo de evolución de la enfermedad baja $(1,9)$ (dato también corroborado en nuestro estudio), ayudaría a entender por qué en estas poblaciones con mayor número de pacientes no se han detectado más hemorragias.

Resulta muy controvertido el análisis de los métodos diagnósticos de la complicación. Algunos estudios han realizado el diagnóstico basándose exclusivamente en los métodos endoscópicos (12), aunque en la mayoría de las series se ha necesitado la combinación de métodos endoscópicos con la arteriografía $(1,11)$. Sería de especial interés, aunque no existan datos en la literatura, valorar la capacidad diagnóstica de la cápsula endoscópica en el diagnóstico de estas hemorragias, especialmente en tramos intestinales altos, donde ha demostrado su eficacia en el diagnóstico de la EC (13). En nuestro medio, la combinación de la endoscopia y la arteriografía ha tenido un alto valor diagnóstico, aunque coincidimos con otros estudios en la escasa capacidad terapéutica de los métodos endoscópicos en estos pacientes con sangrados tan masivos $(1,9)$. Los 3 pacientes de nuestra serie con sangrado digestivo masivo requirieron cirugía, dato que difiere de los reflejados en otras series, en las que el porcentaje de pacientes que precisan resolución quirúrgica del mismo varía entre el 35 y el $65 \%(1,10,12)$. El hecho de presentar todos los casos un origen ileal del sangrado ha dificultado o imposibilitado una resolución endoscópica de la hemorragia aguda, al no haberse alcanzado el punto de sangrado como consecuencia de la gran cantidad de sangre presente en todo el marco colónico. El análisis individual de nuestra serie nos muestra que el 3,4\% de los pacientes con EC que han requerido alguna cirugía en relación con su enfermedad, esta se ha debido a un sangrado masivo. Estas cifras son superiores a otras series, en las que el porcentaje de intervenciones como consecuencia de los sangrados son muy inferiores o solamente anecdóticas $(14,15)$, lo que nos ofrece un dato indirecto del número y la gravedad de las hemorragias.

A pesar de ser una de las complicaciones más graves y potencialmente asociadas a una mayor mortalidad (en series clásicas de hasta el 30\%) (2), en nuestros pacientes, y siguiendo la tendencia de los últimos años $(1,12)$, no se ha observado ningún caso de mortalidad. Probablemente 
la mejoría en las unidades de tratamiento de enfermedades agudas y, en la calidad y condiciones quirúrgicas sean factores a tener en cuenta.

En nuestros pacientes el lugar de sangrado se encuentra localizado en el íleon; y aunque la gran mayoría de las otras series reportadas también refieren la localización del sangrado a ese nivel $(2,9,16)$; se han descritos casos en los que la localización del punto de sangrado es mucho más heterogénea, incluyendo localización colónica $(1,12)$.

El limitado número de pacientes con hemorragias masivas hace muy difícil el análisis y la probable interpretación de los potenciales factores de riesgo. El tabaco se ha descrito en numerosas ocasiones como un indicador de mal pronóstico para la EC y para el desarrollo de sus complicaciones (17), aunque previamente no se ha estudiado como un potencial factor de riesgo para las hemorragias. El hecho de que dos pacientes de nuestra serie fuesen fumadores, a pesar de no ser significativo, nos hace una llamada de atención sobre el nefasto papel que juega el tabaco en la evolución de los pacientes con Crohn.

A pesar de que dos pacientes estaban recibiendo tratamiento con azatioprina, en estudios que han evaluado el uso de azatioprina a largo plazo, no se han referido hemorragias masivas $(18,19)$. Mucho más controvertido es el hecho de que un paciente hubiese requerido infliximab, ya no sólo porque la hemorragia no se ha descrito como una complicación del tratamiento, sino porque se han reportado trabajos en los que casos de hemorragias masivas han sido tratados con resultados muy favorables con infliximab, debido a la rápida cicatrización de la mucosa $(20,21)$.

Los factores genéticos en los últimos años han sido uno de los parámetros más estudiados en cuanto a la presentación y la evolución clínica de la EC (22). En esta misma población hemos descrito cómo la presencia de las mutaciones en NOD2/CARD15 se comportaban como un factor predictivo para la necesidad de cirugía en los pacientes con Crohn (23). Desgraciadamente y, a pesar de que uno de los pacientes sí que presentaba mutaciones genéticas tanto en NOD2 como en TLR4, el escaso número de pacientes, sumado al menor impacto de estas mutaciones en nuestra población en comparación con otras caucásicas europeas (24) hace que no se puedan obtener conclusiones en cuanto al valor predictivo de estas mutaciones en este tipo de pacientes.

En conclusión, la hemorragia digestiva masiva es una complicación poco común, aunque grave de la EC. El diagnóstico es recomendable que se haga tanto endoscópicamente como con arteriografía; como consecuencia de su severidad la resolución suele ser quirúrgica. Son necesarios más estudios y con un número superior de casos para valorar los potenciales factores de riesgo para el desarrollo de esta complicación en estos enfermos.

\section{BIBLIOGRAFÍA}

1. Pardi DS, Loftus EV Jr, Tremaine WJ, Sandborn WJ, Alexander GL, Balm RK, et al. Acute major gastrointestinal hemorrhage in inflam- matory bowel disease. Gastrointest Endosc 1999; 49: 153-7.

2. Robert JR, Sachar DB, Greenstein AJ. Severe gastrointestinal hemorrhage in Crohn's disease. Ann Surg 1991; 213: 207-11.

3. Egawa T, Kuroda T, Ogawa H, Takeda A, Kanazawa S, Harada H, et al. A case of Crohn's disease with recurrent massive life-threatening hemorrhage from terminal ileum. Hepatogastroenterology 1999; 46: 1695-8.

4. McGarrity TJ, Manasse JS, Koch KL, Weidner WA. Crohn's disease and massive lower gastrointestinal bleeding: angiographic appearance and two case reports. Am J Gastroenterol 1987; 82: 1096-9.

5. Gasche C, Scholmerich J, Brynskov J, D'Haens G, Hanauer SB, Irvine EJ, et al. A simple classification of Crohn's disease: Report of Working Party for the World Congresses of Gastroenterology, Vienna 1998. Inflamm Bowel Dis 2000; 6: 8-15.

6. Hugot JP, Chamaillard M, Zouali H, Lesage S, Cézard JP, Belaiche J, et al. Association of NOD2 leucine-rich repeat variants with susceptibility to Crohn's disease. Nature 2001; 411: 599-603.

7. Cario E, Podolsky DK. Differential alteration in intestinal epithelial cell expression of toll-like receptor 3 (TLR3) and TLR4 in inflammatory bowel disease. Infect Immun 2000; 68: 7010-7.

8. Klein W, Tromm A, Griga T, Folwaczny C, Hocke M, Eitner K, et al. A polymorphism in the CD14 gene is associated with Crohn's disease. Scand J Gastroenterol 2002; 37: 189-91.

9. Cirocco WC, Reilly JC, Rusin LC. Life-threatening hemorrhage and exsanguination from Crohn's disease. Report of four cases. Dis Colon Rectum 1995; 38: 85-95.

10. Kostka R, Lukas M. Massive, life-threatening bleeding in Crohn's disease. Acta Chir Belg 2005; 105: 168-74

11. Driver CP, Anderson DN, Keenan RA. Massive intestinal bleeding in association with Crohn's disease. J R Coll Surg Edinb 1996; 41: 152-4.

12. Belaiche J, Louis E, D'Haens G, Cabooter M, Naegels S, De Vos M, et al. Acute lower gastrointestinal bleeding in Crohn's disease: Characteristics of a unique series of 34 patients. Belgian IBD Research Group. Am J Gastroenterol 1999; 94: 2177-81.

13. Gómez Rodríguez BJ, Ortiz Moyano C, Romero Castro R, Caunedo Alvarez A, Hernandez Duran MD, Hergueta Delgado P, et al. Diagnostic yield of 335 push video-enteroscopies. Rev Esp Enferm Dig 2006; 98: 82-92.

14. Farmer RG, Hawk WA, Turnbull RB Jr. Indications for surgery in Crohn's disease: Analysis of 500 cases. Gastroenterology 1976; 71: 245-50.

15. Fazio VW, Wu JS. Surgical therapy for Crohn's disease of the colon and rectum. Surg Clin North Am 1997;77: 197-210.

16. Homan WP, Tang CK, Thorbjarnarson B. Acute massive hemorrhage from intestinal Crohn disease. Report of seven cases and review of the literature. Arch Surg 1976; 111: 901-5.

17. Picco MF, Bayless TM. Tobacco consumption and disease duration are associated with fistulizing and stricturing behaviors in the first 8 years of Crohn's disease. Am J Gastroenterol 2003; 98: 363-8.

18. Colombel JF, Loftus EV Jr, Tremaine WJ, Pemberton JH, Wolff BG, Young-Fadok T, et al. Early postoperative complications are not increased in patients with Crohn's disease treated perioperatively with infliximab or immunosuppressive therapy. Am J Gastroenterol 2004; 99: 878-83.

19. Dubinsky MC. Azathioprine, 6-mercaptopurine in inflammatory bowel disease: Pharmacology, efficacy, and safety. Clin Gastroenterol Hepatol 2004; 2: 731-43.

20. Papi C, Gili L, Tarquini M, Antonelli G, Capurso L. Infliximab for severe recurrent Crohn's disease presenting with massive gastrointestinal hemorrhage. J Clin Gastroenterol 2003; 36: 238-41.

21. Belaiche J, Louis E. Severe lower gastrointestinal bleeding in Crohn's disease: Successful control with infliximab. Am J Gastroenterol 2002; 97: 3210-1.

22. Gómez de La Concha E, Fernández Franco L. Inflammatory bowel diseases. Relationship between genetics and clinical characteristics. Rev Esp Enferm Dig 2003; 95: 524-6.

23. Barreiro M, Núñez C, Domínguez Muñoz JE, Lorenzo A, Barreiro F, Potel J, et al. Association of NOD2/CARD15 mutations with previous surgical procedures in Crohn's disease. Rev Esp Enferm Dig 2005; 97: $547-53$

24. Núñez C, Barreiro M, Domínguez Muñoz JE, Lorenzo A, Zapata C, Peña AS. CARD15 mutations in patients with Crohn's disease in a homogeneous Spanish population. Am J Gastroenterol 2004; 99: 450-6. 